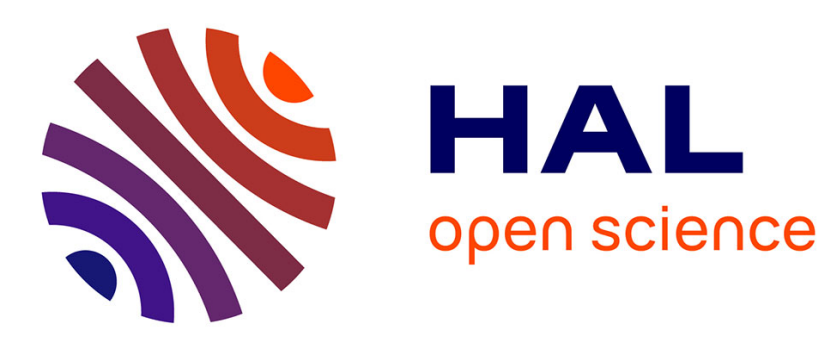

\title{
SAXS study of coated porous silicas
}

\author{
A. Benedetti, S. Ciccariello, F. Pinna, G. Strukul
}

\section{To cite this version:}

A. Benedetti, S. Ciccariello, F. Pinna, G. Strukul. SAXS study of coated porous silicas. Journal de Physique IV Proceedings, 1993, 03 (C8), pp.C8-463-C8-466. 10.1051/jp4:1993896 . jpa-00252327

\section{HAL Id: jpa-00252327 https://hal.science/jpa-00252327}

Submitted on 1 Jan 1993

HAL is a multi-disciplinary open access archive for the deposit and dissemination of scientific research documents, whether they are published or not. The documents may come from teaching and research institutions in France or abroad, or from public or private research centers.
L'archive ouverte pluridisciplinaire HAL, est destinée au dépôt et à la diffusion de documents scientifiques de niveau recherche, publiés ou non, émanant des établissements d'enseignement et de recherche français ou étrangers, des laboratoires publics ou privés. 


\title{
SAXS study of coated porous silicas
}

\section{A. BENEDETTI, S. CICCARIELLO* ${ }^{*}$ F. PINNA ${ }^{* *}$ and G. STRUKUL ${ }^{* *}$}

Dipartimento di Chimica-Fisica, Calle S. Marta DD2137, 30123 Venezia, Italy

${ }^{*}$ Dipartimento di Fisica 'G. Galilei' and sez. INFM, via Marzolo 8, 35131 Padova, Italy

** Dipartimento di Chimica Industriale, Calle S. Marta DD2137, 30123 Venezia, Italy

\begin{abstract}
The small-angle x-ray intensities scattered (SAXS) by four reversed phase silica (RPS) samples are analyzed. The RPS's have been obtained by coating two different porous silica supports with dimethyloctyl- and with dimethyloctadecyl-(chloro)silane. From the observed intensities it is possible to estimate the mean electron-densities of the films as well as the mean silica-surface per coating molecule.
\end{abstract}

\section{1 - Introduction and theoretical model}

It has been recently pointed out that SAXS can be useful for characterizing coatings in porous materials as, e.g., reversed phase silicas. The observed intensities in fact, turn out to be sensitive to the loadings of the samples as well as to the length of the coating molecules[1]. Structural information on the coating films can be obtained by modelling the samples as consisting of three homogeneous phases, the bulk silica, the void regions (pores) and the film of the coating molecules, each characterized by a constant electron density: $n_{1}, n_{2}(=0)$ or $n_{3}$, respectively, the thickness $(\delta)$ of the film region being constant $[2]$. From this model, it follows that the silica-film and the film-void interfaces are parallel. Thus the SAXS intensity, at large $h$ 's, is found to be equal to[3]

$$
I_{l a t}(h)=\frac{2 \pi S \alpha(\nu) n_{12}^{2}}{h^{4}}[1+\mathcal{R}(\nu) \cos (h \delta)]
$$

or to

$$
J_{\text {lat }}(h)=\frac{\pi^{2} S \alpha(\nu) n_{12}^{2}}{h^{3}}\left[1+\mathcal{R}(\nu) \varphi_{0}(h \delta)\right]
$$

depending on the collimation geometry. Here, $S$ denotes the area of silica surface, while $\varphi_{0}(x)$ is a function defined elsewhere[4] and characterized by a damped oscillatory behaviour. The remaining quantities are defined as follows:

$$
n_{i j} \equiv n_{i}-n_{j}, \quad i, j=1,2,3, \quad \nu \equiv\left(n_{13}-n_{32}\right) / n_{12}
$$




$$
\alpha(\nu) \equiv\left(1+\nu^{2}\right) / 2 \quad \text { and } \quad \mathcal{R}(\nu) \equiv\left(1-\nu^{2}\right) /\left(1+\nu^{2}\right)
$$

According to eq.s (1) and (2), a Porod plot of the intensities should show an oscillatory deviation, whose frequency decreases as the length of the coating molecules increases, while its amplitude increases as the sample loading is increased (see eq.s (3b) and (3d)). Figure 1 of this paper as well as figures 1 and 2 of [2] show such a behaviour.

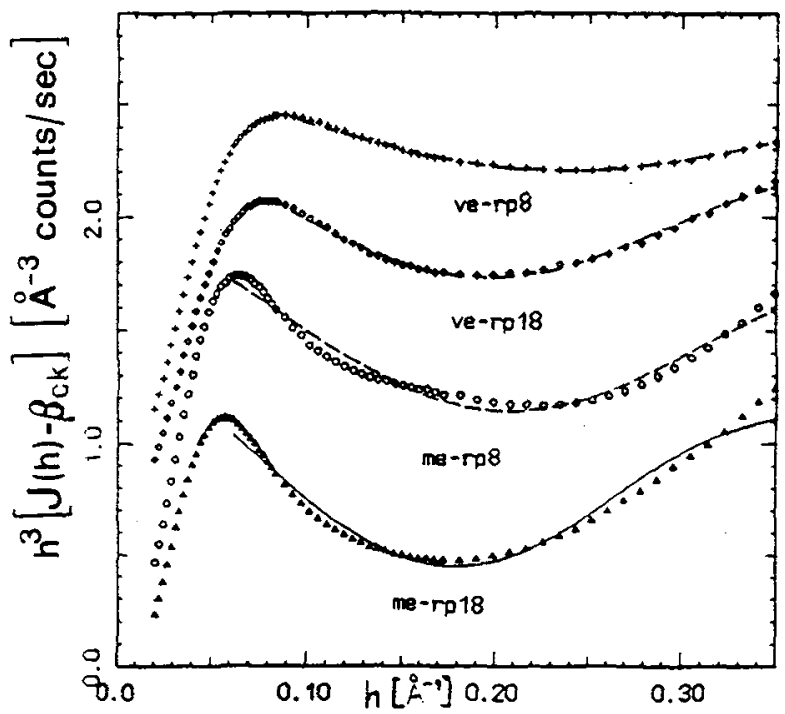

Figure 1. Porod plots of the intensities. Symbols refer to experimental values, while curves represent fitted curves. From the bottom, each curve has been vertically shifted by $0,0.2,0.7$ and 0.9 units, respectively.

Therefore the parameters $\delta, \mathcal{R},\left(\alpha n_{12}^{2} S\right.$ ) and $\beta_{c k}$ (this added parameter represents the constant "Laue background" due to electron density fluctuations on atomic scale[5]) can be estimated by minimizing the quantity

$$
\chi_{s l t}^{2}=\sum_{h_{I} \leq h_{j} \leq h_{F}}\left\{\left[\left(J_{e x p}\left(h_{j}\right)-\beta_{c k}\right)-J_{l a t}\left(h_{j}\right)\right] / J_{w, j}\right\}^{2} .
$$

$J_{\text {exp }}\left(h_{j}\right)$ denotes the 'ideal slit' experimental intensity at the jth momentum transfer value $h_{j}, J_{l a t}\left(h_{j}\right)$ is given by eq. (2) and $J_{\varpi, j} \propto\left[J_{e x p}\left(h_{j}\right)\right]^{\varpi}$, with $\varpi=1 / 2$ or 1 depending on whether the intensities are measured at fixed time or at fixed counts[6]. $h_{I}$ and $h_{F}$ denote the lower and upper bounds of the asymptotic region. The considered $h_{F}$ values correspond to the largest observed scattering angles, while the $h_{I}$ 's are roughly equal to the positions of the (1st) peak in the Porod plots. 


\section{2 - Numerical analysis and results}

The aim of this paper is to better assess the accuracy of the technique by comparing the resulting parameters with the values obtained by chemical analysis. The Table reports the results obtained by best-fitting the intensities relevant to some coated silica samples. The intensities were collected with a Kratky camera equipped with an electronic step scanner[2]. Samples ME-RP8 and ME-RP18 belong to Merck's Lichroprep series obtained by fully derivatizing a silica support, having pores of $100 \dot{A}$, with dimethyloctyl- and with dimethyloctadecyl-chlorosilane, respectively. Samples VE-RP8 and VE-RP18 have been similarly prepared in our laboratory starting from the AKZO Chemie F-22 silica support with pores of $90 \dot{A}$. Therefore, the two sets of fully loaded samples differ only in the geometry of the supports.

Beside the quantities already defined, the remaining quantities in the Table are defined as follows: $\tau \equiv n_{3} / n_{1}, s$ is the support surface area per coating molecule, $\bar{\sigma} \equiv(S /(V \Phi(\nu,\{\phi\}))$ where

$$
\Phi(\nu,\{\phi\}) \equiv\left[\phi_{1} \phi_{2}+(1+\nu)^{2} \phi_{1} \phi_{3} / 4+(1-\nu)^{2} \phi_{2} \phi_{3} / 4\right] .
$$

Finally, $S_{r u}$ and $Q_{P r u}$ denote the film area and the Porod invariant values obtained from the intensity profiles in relative units.

\section{Table}

$\delta$ : film thickness; $\mathcal{R}(\nu)$ : defined by eq. $(3 \mathrm{c}) ; \tau \equiv n_{3} / n_{1} ; \bar{\sigma}$ : defined above eq. $(5) ; s:$ silica surface area per coating molecule; $\beta_{c k}$ : theoretical background; $n_{12}^{2} S_{r u}=\left(n_{1}-n_{2}\right)^{2} S_{r u}$ : proportional to the support surface; $Q_{P_{r u}}$ : Porod invariant obtained from the relative unit intensity; $h_{I}-h_{F}$ : extrema of the best-fit $h$-range; $\bar{\chi} \equiv \chi^{2} /\left(N_{\text {exp }}-N_{p a r}\right)$ : statistical indicator of the fit goodness.

\begin{tabular}{|c|c|c|c|c|c|}
\hline sample & $\delta[\dot{A}]$ & $\mathcal{R}(\nu)$ & $\tau$ & $\bar{\sigma}\left[\dot{A}^{-1}\right]$ & $s\left[\dot{A}^{2}\right]$ \\
\hline ME-RP 8 & $13 \pm 4$ & 0.43 & $0.82 \pm 0.06$ & 0.19 & $13 \pm 5$ \\
\hline ME-RP18 & $15 \pm 2$ & 0.76 & $0.68 \pm 0.05$ & 0.15 & $25 \pm 4$ \\
\hline VE-RP 8 & $11 \pm 6$ & 0.19 & $0.91 \pm 0.15$ & 0.18 & $14 \pm 6$ \\
\hline VE-RP18 & $14 \pm 3$ & 0.34 & $0.85 \pm 0.07$ & 0.18 & $22 \pm 5$ \\
\hline sample & $\beta_{\text {ck }}$ & $n_{12}^{2} S_{r u}$ & $Q_{P r u}$ & $h_{I}-h_{F}\left[\dot{A}^{-1}\right]$ & $\bar{\chi}$ \\
\hline ME-RP 8 & $14 \pm 6$ & $0.19 \pm 0.02$ & 318 & $0.06-0.35$ & 0.78 \\
\hline ME-RP18 & $16 \pm 9$ & $0.15 \pm 0.02$ & 239 & $0.06-0.35$ & 1.87 \\
\hline VE-RP 8 & $31 \pm 7$ & $0.18 \pm 0.04$ & 310 & $0.08-0.35$ & 0.03 \\
\hline VE-RP18 & $24 \pm 6$ & $0.17 \pm 0.02$ & 271 & $0.08-0.35$ & 0.07 \\
\hline
\end{tabular}

Inspection of the resuits allows one to estimate the consistency and accuracy which can be obtained by the aforesaid analysis. Since the analyzed samples have approximately the same volume and have been similarly prepared, $n_{12} S_{r u}, \beta_{c k}$ and $\bar{\sigma}$ should depend mainly on the support. On the contrary, $\delta, s$ and, to a lesser extent, $\tau$ should depend 
mainly on the length of the coating molecules. These properties appear to be reasonably verified.

\section{3 - Further checks and conclusion}

Further checks are possible due to the noted uniformity in sample preparation and to the use of the same experimental apparatus. In this case: $Q_{P r u, l}=C n_{12}^{2} \Phi\left(\nu_{l},\left\{\phi_{l}\right\}\right)$, where the index $l$ labels the different RPS's obtained from the same support. (Note that the constant $C$ is independent of $l$.) It is reasonable to assume that the derivatization of a silica support amounts to leaving $\phi_{1}$ constant and to decreasing $\phi_{2}$ by the film volume fraction $\phi_{3, l} \approx S \delta_{l} / V$. Therefore, when the mass and $Q_{P r u, 0}$ relevant to the silica support sample are known, taking the ratios of the $Q_{P r u, l}$ 's with $Q_{P r s, 0}$ and using the $\nu_{l}$ values resulting from the $\mathcal{R}(\nu)$ 's reported in the table, both $S / V$ and $\phi_{1}$ can be determined. For the AKZO F-22 support $Q_{P r u, 0}=314$ and it results that the specific surface and the void-volume fraction are equal to $600 \mathrm{~m}^{2} / \mathrm{g}$ and 0.66 respectively, while the manufacturer nominal values are $400 \mathrm{~m}^{2} / \mathrm{g}$ and 0.80 .

A further check on the $s$-values can be obtained by measuring the RPSs' carbon content by elemental analysis. The weight percents of carbon were found equal to $8.46 \%$ and to $10.98 \%$ in samples VE-RP8 and VE-RP18, respectively. This corresponds to one coating molecule per 17 and per $29 \mathrm{SiO}_{2}$ molecules, respectively. The support being the same and its void-volume fraction high, the ratio $17 / 29$ must be approximately equal to the ratio of the corresponding $s$-values reported in the table, i.e. 14/22. For the ME-RP8 and ME-RP18 samples, the carbon weight percents are: 9.64 and 17.24, which imply a coating molecule per $18 \mathrm{SiO}_{2}$ molecules in both samples. Thus, the ratio of the corresponding $s$ 's should be one, while the table results give $13 / 25$.

In conclusion, by eq.s (1-3) the analysis of the deviations from the Porod behaviour of the small-angle intensities, scattered by coated porous materials, provides reliable structural information (thickness, electron density, area per coating molecule) on the coating films.

* Author for correspondence.

\section{References}

[1] Schmidt P., Avnir D., Levy D., Höhr A., Steiner M. and Röll A., J. Chem. Phys. 94 (1991) 1474.

2] Benedetti A. and Ciccariello S., DFPD 92/CM/14, submitted to J. Appl. Crystall.

[3] Ciccariello S., J. Appl. Crystall. 24 (1991) 509.

[4] Ciccariello S. and Benedetti A., J. Appl. Crystall. 19 (1986) 195.

[5] Luzzati V., Witz J. and Nicolaieff A., J. Mol. Biol. 3 (1961) 367.

[6] Azaroff L.V., Kaplow R., Kato N., Weiss R.J., Wilson A.J.C. and Young R.A., X-ray diffraction, (Mc Graw Hill, New York, 1974), pp. 482-483. 